\title{
Siete competencias gerenciales Apalancadas por la Inteligencia Emocional: Aplicación de la indagación Apreciativa en una Empresa Colombiana.
}

\author{
Camila Salgado 2
}

Recibido: 02/12/2016 Aceptado: 04/09/2017

DOI: 10.21772/ripo.v35n2a04

\begin{abstract}
Resumen
Se llevó a cabo un proceso de Indagación Apreciativa a lo largo de dos años basado en un modelo de siete competencias gerenciales básicas (autoconciencia y maestría del contexto, automotivación y gestión, autorregulación y empatía, pensamiento estratégico e innovación, comunicación apreciativa y asertiva, negociación contextualizada y manejo de conflictos, actitud colaborativa y construcción de equipos) para TERRANUM, una organización colombiana que maneja finca raíz. La intención era incrementar en cada uno de los 32 directores que integraron el grupo de estudio, siete competencias gerenciales básicas, a partir de su autoevaluación y los resultados de cuatro pruebas. Los gerentes compartían la necesidad de desarrollar aspectos de su inteligencia emocional, personal y social y habilidades relacionadas: autoconciencia personal y contextual, consciencia de sus motivaciones y autorregulación. Los resultados finales mostraron que el $78 \%$ de los participantes en el estudio, incrementaron sus competencias para practicar el liderazgo transformacional, $72 \%$ la comunicación efectiva y $68 \%$ incrementaron su autorregulación emocional.
\end{abstract}

Palabras clave: Inteligencia emocional, Competencias Gerenciales, Gerencia, Habilidades Gerenciales.

\section{Seven Management Competences Supported by Emotional Intelligence: Performing an Appreciative Inquiry in a Colombian Enterprise}

\begin{abstract}
Abstrac
An appreciative inquiry was performed over the course of two years based on a model of seven basic management competencies (self-consciousness and mastery of context, self-motivation and self-management, self-regulation and empathy, strategic thinking and innovation, appreciative and assertive communication, contextualized negotiation and conflict management, cooperative attitude and team building) for TERRANUM, a Colombian organization dealing with real state. The intent was to increase in each of the 32 directors who integrated the study group, seven basic management competencies, based on their self-evaluation and the results of four objective tests. The managers shared the need to develop aspects of their emotional personal and social emotional intelligence competencies and related skills: personal and contextual self-awareness, consciousness of their motivations and self-regulation The final results showed that $78 \%$ of the participants in the study increased their competencies to practice transformational leadership, $72 \%$ effective communication and $68 \%$ increased emotional self-regulation.
\end{abstract}

Keywords: Emotional Intelligence, Management Competencies, Management, Management Abilities.

El enfoque de coaching de la autora es derivado del coaching cognitivo comportamental, la terapia de ese enfoque y una visión sistémica

Nota: El presente artículo fue publicado en el 2017. Sin embargo, con el fin de mantener la periodicidad de la Revista, el número corresponde al segundo semestre del año 2016.

Cómo citar este artículo: Salgado, C. (2016). Siete competencias gerenciales Apalancadas por la Inteligencia Emocional: Aplicación de la indagación Apreciativa en una Empresa Colombiana. Revista Interamericana de Psicología Ocupacional, 35(2), 90-102, Doi: 10.21772/ripo.v35n2a04

2 Ph.D, Consultora según demanda Educación Ejecutiva, Facultad de administración, Universidad de los Andes. Profesora según demanda, Departamento de Psicología Universidad de los Andes. E-mail: camsa12002@gmail.com 


\section{Introducción}

Desde el inicio de la práctica de la autora como Coach $\mathrm{Gerencial}^{3}$, fue posible evidenciar que los directivos que requerían potencializar sus competencias y habilidades, tenían en común la necesidad de desarrollar diversos aspectos de su inteligencia emocional, para ser conscientes de sí mismos, de sus entornos, de sus motivaciones, manejar proactivamente sus emociones y tomar decisiones acertadas.

Uno de los factores que tiene mayor peso en el bienestar integral en las organizaciones y en el grado y la calidad de logro, es el nivel de inteligencia emocional que tengan los directivos y sus colaboradores (Goleman, Boyatzis, Mckee, 2015). En mayor o menor grado, las emociones están presentes en todas las interacciones humanas y en el relacionamiento que requieren los procesos de liderazgo de sí mismo y de otros. Desde el "pienso, luego existo" (Descartes, 1637) hemos llegado a la noción de que "somos seres sintientes que pensamos" (Demasío, 1996); por lo cual, asumir y optimizar los aspectos positivos de nuestra dotación emocional es un reto que solo redunda en beneficios.

El modelo que se desarrolló e implementó durante estos dos años de indagación apreciativa, está íntimamente ligado a la detección y el desarrollo de las competencias personales y sociales de la inteligencia emocional (Goleman, 1998), como se ve en la Figura 1. La intención fue la de potencializar en cada uno de los directivos de Terranum siete competencias gerenciales básicas; teniendo en cuenta no solo su criterio frente a lo que ellos consideraban que necesitaban más sino lo que las pruebas objetivas ${ }^{4}$ que se realizaron mostraban.

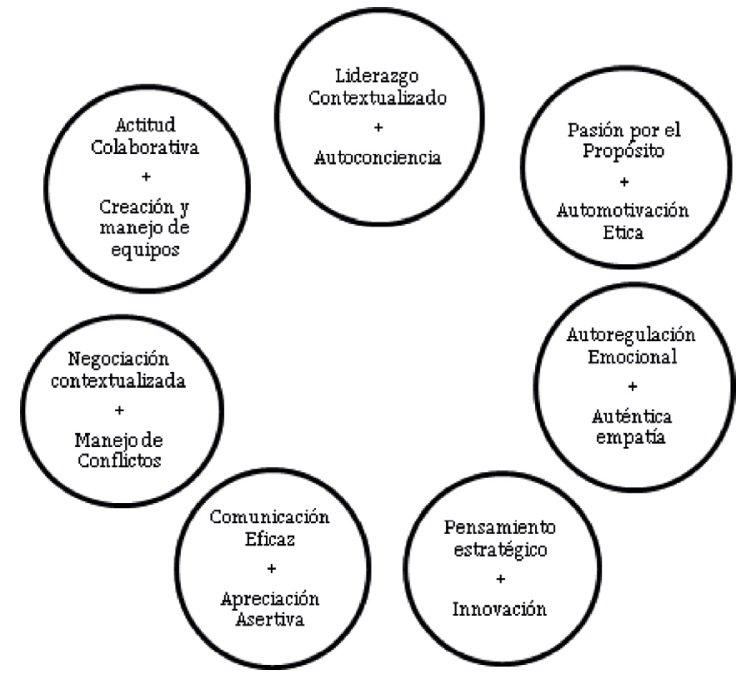

Figura 1: Modelo de las 7 competencias gerenciales apalancadas por la inteligencia emocional (Salgado, 2014).

\footnotetext{
3 El enfoque de coaching de la autora es derivado del coaching cognitivo comportamental, la terapia de ese enfoque y una visión sistémica

4 Pruebas objetivas aplicadas a los participantes 1. Disc / 2. Estilos de Comunicación / 3. Disparadores Emocionales / 4. Estilos de Liderazgo/ 5. Manejo del tiempo
}

\section{Pregunta de Investigación}

¿Cuál es el impacto percibido sobre el desempeño de 32 directivos pertenecientes a la empresa Terranum tras desarrollar 7 competencias personales y sociales de la inteligencia emocional?

\section{Objetivo Principal}

Determinar el impacto de la implementación del modelo para el desarrollo de las siete competencias gerenciales básicas apalancadas por la inteligencia emocional planteado por la autora en el desarrollo de las habilidades gerenciales de los participantes del grupo objetivo de la empresa Terranum, gerentes, líderes y facilitadores.

\section{Objetivos Específicos}

1. Descubrir cuáles son los mayores desafíos de cada participante para su gestión, qué tipo de competencias necesitan desarrollar y por qué.

2. Indagar sobre la interacción de cada participante con su equipo de trabajo para detectar qué competencias estaban aplicando y cuáles requieren potencializar.

3. Lograr que los participantes adquieran herramientas que les permitan auto-conocerse para actuar de acuerdo a una estrategia orientada a su desarrollo dentro del marco de la indagación apreciativa (Kunni, 2003)

\section{Marco Teórico}

\section{Inteligencia Emocional}

La inteligencia emocional es "la capacidad para supervisar los sentimientos y las emociones de uno mismo y de los demás, de discriminar entre ellos y de usar esta información para la orientación de la acción y el pensamiento propio". (Salovey, Peter \& Mayer, 1990, p. 88). Incluye el funcionamiento de un conjunto de componentes y características interrelacionadas; descritas en la Tabla 1.

Tabla 1: Componentes de la inteligencia emocional

\begin{tabular}{clll}
\hline Componente & \multicolumn{1}{c}{ Definición } & \multicolumn{1}{c}{ Distintivos } \\
\hline \multirow{4}{*}{$\begin{array}{l}\text { Autoconcien- } \\
\text { cia }\end{array}$} & $\begin{array}{l}\text { Conocer las emociones, } \\
\text { fortalezas, debilidades, } \\
\text { valores, metas perso- } \\
\text { nales y su impacto en } \\
\text { otros. }\end{array}$ & $\begin{array}{l}\text { Autoconfianza. } \\
\text { Autoevaluación realista. } \\
\text { Autocrítico sentido del } \\
\text { humor. }\end{array}$ \\
\hline \multirow{2}{*}{ Auto } & $\begin{array}{l}\text { Sed de Crítica Construc- } \\
\text { tiva. }\end{array}$ \\
\hline $\begin{array}{l}\text { Controlar las emocio- } \\
\text { nes disruptivas y los } \\
\text { impulsos. }\end{array}$ & $\bullet$ & $\begin{array}{l}\text { Confiabilidad } \\
\text { Integridad } \\
\text { Comodidad con el cam- } \\
\text { bio }\end{array}$ \\
\hline
\end{tabular}




\begin{tabular}{|c|c|c|}
\hline Componente & Definición & Distintivos \\
\hline Motivación & $\begin{array}{l}\text { Impulsar para obtener } \\
\text { logros. }\end{array}$ & $\begin{array}{l}\text { - Pasión por el trabajo y } \\
\text { por los nuevos retos. } \\
\text { Inagotable energía para } \\
\text { mejorar. } \\
\text { Optimismo encarando el } \\
\text { fracaso. }\end{array}$ \\
\hline Empatía & $\begin{array}{lrr}\text { Considerar } & \text { los } \\
\text { sentimientos de } & \text { los } \\
\text { otros, especialmente } \\
\text { cuando se } & \text { toman } \\
\text { decisiones. } & \end{array}$ & $\begin{array}{l}\text { Experiencia en la atrac- } \\
\text { ción y retención de ta- } \\
\text { lento. } \\
\text { Habilidad para desarro- } \\
\text { llar a otros. } \\
\text { Sensibilidad para afron- } \\
\text { tar diferencias cultura- } \\
\text { les. }\end{array}$ \\
\hline $\begin{array}{l}\text { Habilidades } \\
\text { Sociales }\end{array}$ & $\begin{array}{l}\text { Administrar las relacio- } \\
\text { nes, para mover a las } \\
\text { personas a la dirección } \\
\text { deseada. }\end{array}$ & $\begin{array}{l}\text { Eficacia en el cambio de } \\
\text { liderazgo. } \\
\text { - Amplia experiencia en } \\
\text { trabajo en redes. } \\
\text { - Experiencia en construir } \\
\text { y liderar equipos. }\end{array}$ \\
\hline
\end{tabular}

Nota: Tomada de HBR's 10 Must Reads. On emotional intelligence. USA: Harvard Busness Review Press (pags 4- 5, traducido por Camila Salgado)

Goleman, et al., (2015) amplían el concepto original de inteligencia emocional con base en una investigación sobre lo que sucede en el cerebro cuando la gente interactúa. Usan el término de inteligencia social, como una serie de competencias interpersonales que están fundamentadas en circuitos neuronales específicos.

Según los expertos, los grandes líderes estudiados comparten un nivel alto de inteligencia emocional, que les permite ejercer un rol transformacional sobre la organización y sus colaboradores. El soporte del sistema emocional es biológico, este es susceptible de mejorar a partir del aprendizaje y de la práctica deliberada y sistemática de nuevas conductas; es decir, podemos modificar intencionalmente nuestra forma de responder. Cada emoción se caracteriza o está asociada a un tipo de valoración significativa y esta valoración puede no corresponder a lo que está teniendo lugar en el presente, de ahí la importancia de la autoconciencia y la autorregulación emocional.

\section{Autoconsciencia. Pilar del logro gerencial}

\author{
"A tu propio ser se verdadero" \\ Shakespeare (1564-1616) \\ "Solamente hay un rincón del universo que con certeza puedes \\ mejorar: y ese es tu propio ser" \\ Aldous Huxley (1894-1963)
}

La autoconciencia es la primera de las tres competencias personales que hacen parte de la inteligencia emocional. Es el pilar de la inteligencia emocional; la condición más importante para el autoconocimiento, la toma de decisiones y el desarrollo de acciones efectivas. Nos permite ubicarnos en el presente, ser conscientes de nosotros mismos, de lo que sucede en nuestro interior, ser conscientes de otros seres y del entorno. Autores como Goleman (2015), Boyatzis (2015), y McKee (2015) la consideran piedra angular del liderazgo de sí mismo y de otros. Implica autoconocimiento de fortalezas, vulnerabilidades, oportunidades de mejora e intereses (Rath, 2007). Implica el reconocimiento de nuestro medio y la capacidad de contextualizarnos a su naturaleza y desafíos. Exige asumir que existen "realidades" distintas a nuestras expectativas a las cuales conviene adaptarnos siempre y cuando concuerden con nuestros valores prioritarios.

La autoconciencia abarca dos dimensiones; la conciencia cognitiva que permite atender y focalizarnos en aquello que es el objeto de nuestro interés, sumada a la conciencia emocional de otros y del entorno. La persona autoconsciente responde de acuerdo a las características y exigencias particulares del contexto en el cual tiene lugar la interacción, considerando, por ejemplo, las diferencias interculturales o sociodemográficas de los interlocutores, como lo hace el camaleón, mimetizándose con el entorno sin dejar de ser un camaleón. Un nivel alto de autoconsciencia, es característico de los grandes líderes y de los emprendedores exitosos (Bill George, 2007).

\section{Automotivación}

La automotivación es el "motor" para pensar, sentir y actuar ya sea de forma positiva o negativa (Goleman, 2006). Puede ser positiva si está contextualizada y fundamentada o negativa en caso contrario. Emerge de nuestra condición psicofisiológica; de nuestras experiencias, aprendizajes, sistema de creencias, nivel de energía y hábitos de autocuidado. Está ligada a la ética y a nuestros valores no negociables. La pasión por el propósito es el energizante más poderoso para el logro del objetivo propuesto. (McChesney, C., Covey, S., \& Huling, J, 2016).

La competencia de automotivación nos permite, a través de la autoconciencia y el manejo del contenido del pensamiento, orientarnos hacia el logro o hacia el fracaso. No en vano se habla del poder del pensamiento positivo. Las cosas no son como son sino como imaginamos que son. (Anais Nin, 1961). Autores como Beck (2014) y Covey (2016), muestran cómo la automotivación positiva está ligada al pensamiento optimista, fundamentado en elementos reales (Beck, 2004). Si la persona tiene un optimismo que es más fantasioso, se arriesga a fracasar. Así, un grado moderado de "pesimismo" permite contemplar y anticipar los riesgos de las acciones y decisiones (Cantor, Norem et. Al, 1987). Este hecho se relaciona con la psicología del autoengaño (Mertz, 2004), que ejercen aquellos optimistas sin fundamento para serlo. (Goleman, 1998). 
La automotivación implica autogestión en situaciones de desafío y la disposición para ver opciones de solución. Está ligada con nuestra predisposición genética hacia el optimismo y el desarrollo de nuestra capacidad de discernir el impacto que pueden tener nuestras expectativas no conscientes en nuestros objetivos (McChesney, C., Covey, S., \& Huling, J, 2016). En la actualidad modelos sólidos como el cognitivo comportamental resaltan la relación entre pensamiento y acción, lo cual es muy antiguo. Según Beck (1979) no es la situación lo que afecta emocionalmente a las personas sino más bien sus pensamientos en esa situación. Pensamientos anticipatorios de tipo negativo o catastrófico, generan un estado de malestar que impide pensar de forma clara y realista, tal como podemos hacerlo cuando estamos emocionalmente balanceados, cuando nuestras emociones corresponden a la naturaleza de la situación presente.

\section{Autorregulación}

Esta competencia nos permite detectar nuestro impulso a reaccionar y por lo tanto, controlar su forma de manifestación (Fisher \& Shapiro, 2005). El autoconocimiento derivado de la autoconsciencia es lo que facilita la autorregulación, a partir de detectar en nosotros mismos y en los demás las señales corporales que acompañan a la emoción y los pensamientos asociados.

Para poder autorregular nuestras emociones, es indispensable ser conscientes de nuestras vulnerabilidades, de nuestro "Talón de Aquiles", frente a situaciones que nos “disparan", lo cual nos permitirá ser proactivos en vez de reactivos. Pruebas como la de Talent Smart (2010) y la de disparadores emocionales usada en el estudio, facilitan el incremento de la competencia de autorregulación (Salgado, 2013).

El control voluntario de la conducta involuntaria impulsada por las emociones no es fácil, especialmente si las emociones que se sienten son intensas. Podemos detener nuestro discurso y acciones, más que detener cualquier indicio de la emoción en nuestra cara o voz. Las culturas orientales afirman que una forma básica para incrementar nuestra consciencia, es el "estar atento". Significa estar en la situación presente, saber cuándo estamos dominados por las emociones, cuándo podemos perder el control sobre lo que hacemos o decimos y luego podríamos lamentar.

\section{Autogestión}

Auto gestionarse es hacerse cargo de sí mismo. Santiago Ramón y Cajal, premio Nobel de Medicina en 1906, dijo: "Todo ser humano, si se lo propone, puede ser escultor de su propio cerebro". La autogestión se apoya en la interrelación de las tres competencias personales de la inteligencia emocional (Tabla 1). Es el producto de sumar la autoconciencia y la automotivación para lograr el grado de autorregulación personal que permite pensar antes de actuar, fijarse una meta prioritaria de desarrollo, focalizarse en ella, y tener la disciplina, la perseverancia y la tolerancia a la frustración para lograr lo propuesto.

Hay que tomar consciencia de que estamos construyendo nuestro cerebro y posibilidades de bienestar o malestar a partir de acciones, pensamientos y respuestas emocionales (Martin, 2013).

A partir del desarrollo de la autogestión, producto de la suma de la autoconsciencia, la automotivación y la autorregulación, estamos preparados para conectarnos afectiva y efectivamente con nosotros mismos, con los demás y poner en acción las competencias sociales de la inteligencia emocional que son la empatía y la gestión de relaciones, indispensables para desempeñarse a nivel personal y gerencial.

\section{Empatía Auténtica}

Para que las manifestaciones de la empatía sean efectivas deben ser congruentes con lo que se siente de fondo. La empatía auténtica exige respeto por la diferencia y sensibilidad intercultural. Es la capacidad de ponerse en el lugar del otro sin absorber lo del otro. (Waytz, 2016). Implica habilidad para sincronizarse con el interlocutor, la capacidad para detectar y satisfacer sus intereses centrales (Goleman, 2009). La empatía auténtica tiene dos componentes: uno cognitivo, muy relacionado con la capacidad para abstraer los procesos mentales de otras personas y otro emocional, que sería la reacción ante el estado emocional percibido (Goleman, 2013).

Para poder experimentar empatía, tenemos que tener, ante todo, consciencia emocional de la existencia y "realidad de las demás personas". Ésta actitud nos facilita observar lo que sienten y expresan otros; además, permite que nos comportemos de forma empática. Según Goleman (2009) existen tres tipos de empatía:

Cognitiva. Nos permite conocer la manera de pensar de otros y responderles en esos términos. Los buenos comerciales la tienen muy desarrollada.

Emocional. Permite detectar y sentir lo que experimenta la otra persona para responder de forma que se sienta tenida en cuenta.

Compasiva. No solo entiende la forma de pensar del otro, sino que se conecta con su emoción y responde apropiadamente; incluso, actúa de manera efectiva para ayudar a la persona. Este último tipo de empatía, es la que se da en una persona con una auténtica y profunda orientación hacia el servicio. 
La empatía se da a través de la comunicación. Implica la capacidad tanto de leer el lenguaje no verbal de los demás, como de manejar un estilo de comunicación clara y asertiva. El dominio de las competencias emocionales personales, sumado a la empatía, permite co-crear un contexto apropiado para desarrollar relaciones eficaces que fomenten el crecimiento personal y la productividad excepcional.

\section{Pensamiento Estratégico}

Autores como Aron and Simerson (2015), Montgomery (2012), Simons (2010) y Porter (1995), tienen una visión de la ciencia y el arte de la gerencia, en la cual el énfasis recae en el pensamiento estratégico, asociado a la capacidad de liderar y la toma de decisiones acertadas. Para pensar estratégicamente hay condiciones previas tales como la capacidad de ubicarse en el presente, ser consciente de sí mismo, del entorno, de las características de la organización y tener la automotivación ética y pertinente que genera pasión por lo que se hace.

El pensamiento estratégico es definido por varios de los autores mencionados como un proceso mental o de pensamiento aplicado por un individuo para lograr éxito sea en un juego o en otro emprendimiento. Im de la estrategia, en la cual A. Ruelas, ( 2012) ticulo onceptualmente, de forma imaginativa, sistematica y oportuna, -nido yplica capacidad de usar procesos conceptuales que faciliten una visión futura de los pasos a seguir para el logro de una meta, poniendo en marcha un conjunto de habilidades críticas que permiten desarrollar un negocio claramente definido y focalizado. El pensamiento estratégico se enfoca en desarrollar oportunidades para crear valor y en encontrar los mejores medios para llegar a la meta. Igualmente, puede definirse como la capacidad del individuo para pensar conceptualmente, de forma imaginativa, sistemática y oportuna.

\section{Comunicación Eficaz}

Un conjunto de autores e investigadores coinciden en que la comunicación eficaz es un elemento central en los procesos de interacción humana en distintos niveles incluyendo el laboral (Waslawich 2002; Salgado 2002; Sorensen \& Cooperrider, 2000; Whetten \& Cameron 2011) lo cual es señalado también en los escritos de Goleman (1998) y Cialdini, R, (2011).

Al analizar el proceso de comunicación eficaz se puede observar que se trata de un proceso de causalidad recíproca. Lo anterior, implica que todas las personas que participan en una interacción comunicacional son responsables del curso que ésta tome. Entonces, existen dos niveles simultáneos en la comunicación: el verbal y el no verbal, los cuales requieren ser congruentes entre sí. Lo anterior, a su vez depende del grado de inteligencia emocional del interlocutor, la capacidad de "leer empáticamente" a su colaborador y de enviarle señales no verbales que sean congruentes con lo que se desea expresar. Aspectos como el tono, el ritmo, la intensidad de la voz y la postura corporal que adoptamos al comunicarnos, denotan las emociones que estamos experimentando a nivel no consciente y consciente y esto, a su vez es percibido por el otro.

El cómo nos comuniquemos está íntimamente relacionado con el desarrollo de las competencias de la inteligencia emocional; especialmente, la autoconciencia, la autorregulación y la empatía. Ésta última competencia requiere consciencia integral de que existen otro(s) distintos e igualmente respetables; lo cual nos permite poder ponernos en el lugar del otro, lo que incluye contextualización cultural (Mayer, 2015, p 107). Así, necesitamos conocer tanto los conceptos adecuados como los prejuicios que nos hemos formado alrededor de una persona o de un tema específico.

En síntesis, podemos concluir que, la teoría de la comunicación efectiva sugiere la necesidad de que ésta sea emocionalmente inteligente, que se dé el "estar presente" en el contexto de relación con el otro, en el aquí y en el ahora, con autoconsciencia de las motivaciones y los estados emocionales que surgen conscientemente o de forma automática durante el proceso para tener herramientas que permitan adecuar la comunicación momento a momento.

\section{Negociación y Manejo de Conflictos}

Negociar es un proceso indispensable para la convivencia social. Sirve para resolver las inevitables diferencias que surgen en las relaciones, buscando un acuerdo mutuo a través del diálogo. No se trata de ganar a como dé lugar sino de establecer relaciones basadas en un acuerdo recíproco, capaz de satisfacer las necesidades e intereses de todas las partes involucradas. La idea es negociar para entablar relaciones a mediano o largo plazo, donde ambas partes puedan situarse en un escenario de beneficio mutuo.

Al ser la negociación un proceso donde pueden interactuar dos o más interlocutores, se hace necesario focalizarse en conocer elementos del estilo de personalidad; los cuales, se pueden asociar a un estilo de negociación. Es importante mencionar que identificar estos aspectos facilita saber cómo abordarlos. Igualmente, el negociador requiere capacidad de adoptar el estilo de negociación que más convenga al contexto de la negociación u optar por llevar a alguien que pueda hacerlo.

Posibles Fuentes de Conflicto en la Negociación. Las diferencias que existen entre los intereses centrales de los interlocutores y sus estilos de negociación, pueden hacer que 
se generen desacuerdos que conllevan a escalar a conflictos de diverso grado de intensidad y entorpecer el avance del proceso (Fischer \& Shapiro, 1991-2005). El conflicto puede definirse como "la interacción de gente interdependiente que percibe metas incompatibles e interferencia por parte de cada uno en el logro de las mismas" (Folger, Poole, \& Stutman, 1997). Los desacuerdos surgen de diferencias cognitivas de opinión, que bien manejadas, pueden ser enriquecedoras o transformarse en conflictos en los cuales la emocionalidad prima sobre la razón y los protagonistas pierden la perspectiva estratégica (Beck, 1979) que les permiten "subir al balcón" (Ury, 2008) para evitar tomar como personal lo que expresa el otro de una forma confrontativa.

\section{Generación y Manejo de Equipos: Actitud Colaborativa}

El poder crear y liderar equipos efectivos, sean éstos permanentes o temporales, pero de alto rendimiento, es una habilidad muy importante para cualquier directivo. Los equipos además de la experticia en el tema, requieren una dosis significativa de inteligencia emocional para valorar las diferencias, integrarse y pasar de la actitud competitiva a la colaborativa. Autores como Vanessa Urch y Steven Wolff muestran cómo es importante aprender a utilizar la inteligencia emocional individual, la del grupo y la de los demás grupos con los que se deben interactuar.

De acuerdo a Whetten \& Cameron (2008) la metáfora descriptiva de un equipo funcional es la de los patos canadienses que vuelan en formación de $\mathrm{V}$, manteniendo cada uno su rol, volando según las normas inherentes al equipo y en función de ayudarse los unos a los otros para lograr la meta. Así pues, como dicen los autores, la eficiencia de los equipos efectivos implica los esfuerzos interactivos coordinados de sus miembros y los resultados superan a cualquier ejecución individual excelente.

En resumen, todo equipo necesita un líder inspirador y eficaz que logre ante todo credibilidad dentro de los miembros de su equipo y para que posteriormente pueda articular metas SMART y EVEREST para el equipo. El líder naturalmente requiere manejar las mejores estrategias y prácticas de comunicación para saber autorregularse, tener otras competencias como son credibilidad y articulación de metas, dos capacidades centrales para el éxito de los equipos que un líder esté guiando.

\section{Acerca de Terranum}

La idea del grupo Terranum nació en el año 2004 al interior de una firma de banca de inversión conformada por cuatro socios. La premisa que soportaba la idea, era la oportunidad que existía para crear un vehículo institucional y así, invertir en el sector inmobiliario comercial buscando ser el aliado de las compañías que requerían suplir necesidades inmobiliarias. Grupo Terranum quería convertirse en un puente entre el mercado de capitales y el sector inmobiliario. Para ello, desde el inicio definió los siguientes objetivos para lograr su propósito: Atraer y retener el mejor talento humano, tener socios de primer nivel que aporten conocimiento/capital y montar un esquema de gobierno corporativo bajo los más altos estándares internacionales para atraer grandes inversionistas institucionales.

Actualmente, Terranum tiene activos bajo manejo por $\$ 4$ billones de pesos, lo que la convierte en el líder del sector, así mismo en cuestión de 8 años pasó de 5 a 330 empleados. Adicionalmente, ha recibido premios tales como:

- Premio Fiabci a la excelencia inmobiliaria en la categoría Proyectos Especiales - Oficinas.

- Nominación a Premios Portafolio por la gestión del recurso humano.

- Certificación ISO 90001 para Terranum Administración.

- Euromoney 2015.

- Mejor administrador de inversiones inmobiliarias.

- Mejor desarrollador de oficinas corporativas.

El concepto del Grupo Terranum proviene de El THEATRUM ORBIS TERRANUM considerado el primer atlas moderno elaborado por Abraham Ortelius en 1572. Su símbolo es el ASTROLABIO, un instrumento que permitía a los navegantes saber la hora y determinar la latitud a partir de la posición de las estrellas. El Astrolabio simboliza a la perfección la propuesta de valor del Grupo Terranum pues representa experticia, capacidad para leer el entorno, conocimiento para determinar el rumbo y confiabilidad para llegar a buen puerto.

\section{¿Qué es la Indagación Apreciativa?}

Para desarrollar esta investigación de tipo cualitativo se utilizó una metodología de Indagación Apreciativa, definida como un proceso de desarrollo organizacional enfocado en resaltar y promover las fortalezas individuales y colectivas de una organización para co-crear un clima organizacional inspirador, orientado hacia lo positivo (Hammond S.A, 2013).

\section{La indagación apreciativa parte de los siguientes principios (Cooperrider, 2003):}

\section{El principio construccionista:}

- Nuestra realidad y nuestra identidad son co-creadas.

- No existen verdades absolutas.

- Vemos las cosas como somos, no como son. 
- Todos estamos profundamente interconectados.

- El Lenguaje construye realidades

\section{El principio poético:}

- La vida es una experiencia enriquecedora.

- Vemos lo que queremos ver.

- Es fundamental encontrar lo que queremos potencializar, no enfocarnos lo que queremos eliminar.

- Hay que desarrollar una visión apreciativa.

\section{El principio de simultaneidad:}

- Vivimos en un mundo que creamos con nuestras preguntas.

- El cambio empieza en el momento que nos cuestionamos.

- Las preguntas positivas llevan a la transformación

- El principio Anticipatorio:

- Las imágenes positivas crean futuros positivos.

- Nuestra visión dicta nuestro destino.

- Creamos lo que creemos.

- Los grandes cambios comienzan con pequeños pasos.

\section{El principio Positivo:}

- Las emociones positivas abren nuestra mente.

- Son fundamentales la afirmación y la apreciación.

- Es de suma importancia identificar y promover las fortalezas individuales y colectivas dentro de una organización.

- A través de un enfoque apreciativo basado en los principios anteriores, se busca lograr que los participantes recuerden y resalten sus fortalezas y éxitos profesionales, creando un clima positivo que incentiva la autoconfianza y el compromiso con el crecimiento personal y organizacional.

\section{Metodología}

\section{Participantes}

La población objetivo de este estudio son 32 ejecutivos de una empresa colombiana del sector inmobiliario. Los participantes fueron informados del objetivo del estudio y se obtuvo el consentimiento informado para su participación.

\section{Diseño}

Para desarrollar esta investigación se utilizó un enfoque de indagación apreciativa con el fin de identificar el mejor camino para el éxito personal y profesional de cada participante, encontrando sus fortalezas individuales a través del cuestionamiento y la observación. A lo largo de dos años y siete sesiones divididas en 4 semestres, se recorrió con los participantes las distintas etapas de este proceso (Kessler, 2013):

Descubrimiento (Primer semestre). Utilizando preguntas estratégicas, se motiva a los participantes para que descubran sus fortalezas y recuerden sus logros.

Sueños (Segundo Semestre). Se pide a los participantes que visualicen una situación ideal en la que se cumplan todas sus aspiraciones.

Diseño (Tercer Semestre). Los participantes crean planes y propuestas concretas para desarrollar sus competencias gerenciales a través de la inteligencia emocional.

Destino (Cuarto Semestre). Los participantes implementan las estrategias que co-crearon en la etapa de diseño, se hace seguimiento periódico de sus logros.

El sitio en el cual tuvo lugar el proceso de coaching gerencial y se obtuvieron los resultados que se registran posteriormente, fue en las oficinas de la empresa TERRANUM. Siguiendo el modelo de indagación apreciativa se buscó establecer relaciones con los participantes con el fin de apoyar su proceso de visualización y co-creación de metas desde un enfoque positivo.

Cada participante tuvo tres sesiones individuales a partir del establecimiento personalizado de sus metas de desarrollo, dos sesiones de observación de su interacción con su equipo y dos sesiones individuales de retroalimentación a su relacionamiento y desempeño liderando su equipo. La metodología tuvo en cuenta los cuatro pilares de Terranum: Integralidad, Dinamismo, Rigurosidad y Sostenibilidad.

\section{Descripción de las Fases del Modelo de Intervención}

El modelo consta de siete sesiones distribuidas de la siguiente manera:

1. Se hizo el diagnóstico de fortalezas y logros junto con una visualización inicial para el desarrollo de competencias personales y laborales: en este diagnóstico se realizaron tres entrevistas individuales y se aplicaron las pruebas mencionadas más adelante.

2. Se hizo una primera observación del desempeño de los participantes trabajando con su equipo.

3. Se retroalimentó al participante con respecto al desempeño con su equipo. Con base en lo anterior, el 
participante eligió utilizar herramientas específicas para potencializar el desempeño.

4. Se hizo una segunda observación del desempeño del participante trabajando con su equipo. De acuerdo a esta nueva observación, el participante se fijó en nuevas formas de comportamiento basadas en sus fortalezas individuales.

5. La segunda retroalimentación se realizó basada en el desempeño del participante liderando su equipo. Se validan las conductas efectivas y se apoya la adopción y co-creación de nuevas herramientas.

6. Sesión de cierre y recomendaciones. Establecimiento de metas prioritarias para continuar el proceso de desarrollo.

7. Sesión individual de seguimiento para evaluar el proceso de adopción de nuevas conductas y establecimiento de otras dos metas prioritarias.

Para desarrollar las competencias requeridas por cada uno de los participantes, se utilizó la guía de Talent Smart (2010) diseñada para ese propósito, la cual facilita que el participante aprenda a prestar atención, a focalizarse en lo que está haciendo y a tener una visión que le permita estar contextualizado con el entorno.

A través de un riguroso ejercicio de autoevaluación, los participantes identificaron las siguientes necesidades:

- Autorregulación emocional.

- Comunicación efectiva; emocionalmente inteligente.

- Liderazgo transformacional.

- Manejo de equipos y reuniones efectivas.

- Relación entre patrones familiares internalizados y tendencias de reacción emocional.

Se utilizaron en todos los casos las siguientes lecturas de apoyo:

- "Cómo aplicar las competencias emocionales para manejar las presiones de la vida laboral" (Salgado, 2012)

- "Como manejar estados emociones perturbadores" (Salgado, 2014)

- "Guía para una comunicación eficaz" (Salgado, 2014)

• "How Successful People Think" (Maxwell, 2009)
- "Anotaciones sobre como predisponerse hacia el logro" (Salgado, 2008-2014)

\section{Descripción de las Herramientas Utilizadas}

Se eligieron y utilizaron las siguientes herramientas de evaluación:

- Pruebas de auto reporte, lecturas y videos especializados para facilitar el desarrollo personal y laboral de los participantes.

- Coaching personalizado basado en el modelo de intervención que integra el diagnóstico y desarrollo de la capacidad de Autoconsciencia, Automotivación, Autorregulación Emocional, Comunicación Empática, hábitos de resiliencia y persistencia, para lograr el tránsito de la estrategia a la ejecución y el desarrollo de la actitud colaborativa y manejo de equipos.

- Se verifica el resultado con una encuesta sobre el cumplimiento de las metas propuestas al inicio del proceso.

- Se firma un contrato de Coaching para desarrollo de competencias Gerenciales.

- Se completa la Prueba DISC, que permite determinar un estilo de personalidad o perfil basado en cuatro dimensiones: Dominancia, Influencia, Serenidad (respuesta a los cambios), y Cumplimiento (rigurosidad). El perfil permite determinar cómo se comunica la persona, evalúa cómo actuamos en diferentes entornos, situaciones o frente a otros (People Performance International, 2015); ya que, las personas actúan y reaccionan de acuerdo a la combinación de los cuatro factores.

- Se aplica la Prueba de Estilos de Comunicación (Murphy, 2005) que arroja el estilo de comunicación predominante de una persona, siendo estos: analítico, intuitivo, funcional y personal. En cada estilo se indican cuáles son los aspectos característicos del mismo.

- Se aplica el cuestionario de auto reporte para detectar disparadores emocionales buscando descubrir cuáles son los factores subyacentes que disparan emociones negativas en las personas. De igual forma, les permite crear consciencia de cómo expresan sus emociones y en qué contextos; y cómo en el ámbito laboral requieren desarrollar nuevas competencias para construir mejores relaciones.

- Se aplica el Test Manejo del tiempo (Mind Tools, 2012). Sirve para identificar los aspectos en los que la persona necesita mejorar con base a sus prioridades. De acuerdo a los resultados, se le brindan herramientas 
específicas al participante que permiten un trabajo más eficiente.

- Se llena el cuestionario de Seguimiento de Logro de Metas de los participantes en el proceso; de acuerdo a las metas iniciales planteadas por los participantes (mínimo 1 máximo 3) se creó un cuadro que se revisaba cada quince (15) días en las sesiones de observación de equipo y de esta forma se ajustaban las tareas. Un mes después de terminado el proceso, se realizó una entrevista semiestructurada a los participantes y éstos, autoevaluaron el porcentaje en el cual consideraban habían cumplido las metas propuestas.

- Lecturas: Todos los participantes hicieron las lecturas y expresaron que les fueron muy útiles.

- Seguimiento con el director de la empresa: En mayo de 2017 se llevó a cabo un seguimiento para establecer la relación entre el apalancamiento de las 7 competencias gerenciales y el desarrollo de la inteligencia emocional en los participantes a mediano plazo. Se pidió al director de la empresa, quien había escogido a los 32 directivos para participar en el estudio, que describiera la evolución de los participantes con respecto a competencias gerenciales que ellos habían escogido mejorar: Autorregulación emocional, Comunicación Eficaz y emocionalmente inteligente, Liderazgo transformacional, Manejo de equipos y reuniones efectivas, y relación entre patrones familiares internalizados y tendencias de reacción emocional.

\section{Resultados}

El proceso individual de entrevista de seguimiento que tuvo lugar al mes de concluido el proceso permitió conocer el cumplimiento de las metas que cada participante fijó de acuerdo a las recomendaciones de Recursos Humanos, su visualización y los resultados de las pruebas. A los dos meses de concluir el proceso se realizó una entrevista de seguimiento con el director de la empresa. La observación de los participantes se enfocó en su trabajo con el equipo e indicó que 30 de los 32 participantes había cumplido satisfactoriamente su objetivo e incluso, uno de los participantes al finalizar la segunda y última sesión de observación de su interacción con su equipo, espontáneamente les solicitó que dijeran cómo percibían en ese momento su relacionamiento y abordaje de los objetivos a lograr. Estos le expresaron que habían percibido que había mejorado significativamente.

El siguiente gráfico muestra las metas que los participantes se propusieron al inicio de la intervención y el porcentaje de cumplimiento de éstas cuando se les hizo la entrevista de seguimiento, al mes de concluir el proceso de coaching. El porcentaje de cumplimiento se basa en el auto reporte y en las observaciones del director de la empresa.

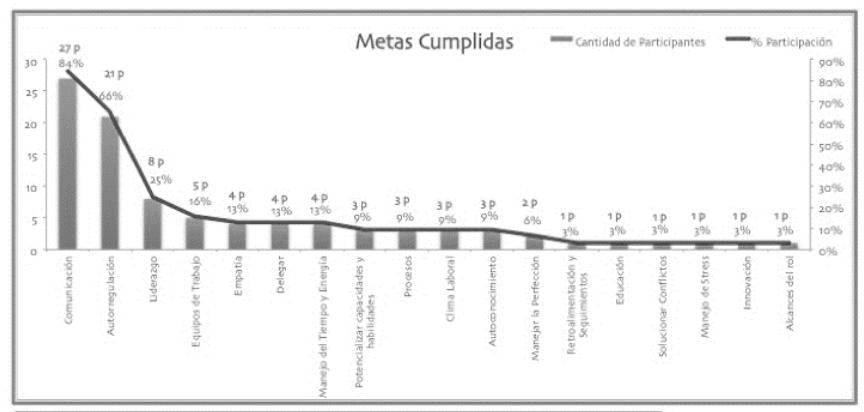

Cantidad Total de Participantes en Proceso $=32$ participantes

Figura 2: Metas cumplidas por los participantes

De acuerdo a los resultados logrados, es posible afirmar que el entrenamiento (siguiendo modelo de competencias propuesto) sí respondió al desarrollo de las habilidades gerenciales que requerían y deseaban potencializar los directivos desde el inicio del proceso.

Se realizaron conversaciones semiestructuradas con el presidente de la compañía y con la directora de gestión humana con respecto a su percepción sobre el efecto del proceso de coaching en los resultados de la gestión de los directivos involucrados. Lo anterior, indicó que estos han optimizado su capacidad de incidir positivamente en sus colaboradores, han facilitado que estos se comprometan con las metas y el esfuerzo que estas implican; de igual modo, adaptarse a algunas reestructuraciones que ha tenido la compañía. Incluso, en varios encuentros con los participantes que terminaron el proceso se evidenció interés por implementar una nueva fase para seguir su desarrollo.

Por otra parte, los participantes han realizado una buena gestión ya que, como se puede demostrar con este informe, han tenido un gran avance en las metas que cada uno se propuso. Las cuales están por encima del $50 \%$ de cumplimiento hasta la fecha. Las áreas que más se han propuesto mejorar son comunicación, autorregulación, liderazgo y en un menor porcentaje, su efectividad con los equipos de trabajo, desarrollo de empatía, capacidad de delegar y un mejor manejo del tiempo y la energía. Los principales sentimientos que se despertaron en los participantes fueron el poder controlar de mejor manera las áreas antes mencionadas (tranquilidad, disminución de estrés, mejoramiento de la calidad de vida, mayor equilibrio entre lo personal y lo laboral, crecimiento en lo personal y lo profesional, mejoramiento en el clima laboral y el trabajo en equipo). No obstante, los participantes consideraron que deben seguir mejorando porque la mayoría no ha llegado al $100 \%$ del desarrollo de su visión. Además, algunos han mencionado la posibilidad de reforzar el conocimiento adquirido con libros, videos y estudios relacionados con 
los temas del coaching que les ayuden a potencializar sus habilidades y las de su equipo.

Un factor muy positivo es que todos son conscientes de la importancia del modelaje como factor definitivo para inspirar a los colaboradores y hacer del contexto de Terranum un semillero de talento. Se trata de desarrollar un modelo de liderazgo que combine la experticia con la co- creación de un ambiente en el cual surja el talento y el bienestar emocional, en el cual los colaboradores sean conscientes de los beneficios personales y profesionales que logran al alinearse con los principios y metas estratégicas de la compañía.

Para determinar el impacto de la intervención a mediano plazo, se llevó a cabo una última sesión de seguimiento con el director (CEO) de la empresa para hablar de los avances que éste ha observado en sí mismo y en el resto de los participantes del estudio:

De los 32 participantes, 9 salieron de la compañía, voluntariamente, por cambiar de ciudad y por una reestructuración estratégica de la misma. Todos los participantes reconocieron la oportunidad de participar en el proceso de coaching para apalancar sus competencias gerenciales como un valor agregado para su desempeño gerencial. El CEO, quien también participó en el proceso, concuerda en la autoevaluación que los gerentes hicieron respecto al cumplimiento de sus metas. El director de la compañía refiere que a raíz de este proceso incrementó la afiliación a la empresa y que el coaching contribuyó a manejar positivamente el proceso de reorganización institucional.

El foco de Terranum en el desarrollo de su capital humano es ampliamente reconocido. El proceso de coaching gerencial ha sido congruente con la misión y visión de la compañía.

El que la autopercepción de los participantes coincida con la del CEO director del equipo, valida la afirmación de la gran mayoría que dice haber incrementado su autoconsciencia. $\mathrm{Su}$ adaptación al cambio y al desafío de la reorganización concuerda con el desarrollo de la autorregulación emocional y la capacidad de liderazgo emocionalmente inteligente.

\section{Conclusiones y Recomendaciones.}

Fue posible observar desde el punto de vista la indagación apreciativa que el proceso permitió que los participantes descubrieran sus fortalezas a través de la autoconciencia y se percataran de las competencias que requerían optimizar para poder actuar como gerentes integrales, capaces de liderar eficazmente y aplicar los principios del coaching para facilitar el desarrollo de sus colaboradores. Lograron asumir y experimentar el inmenso papel que juegan las emociones en la vida personal y laboral, focalizándose en desarrollar un clima emocional positivo, que facilitara la productividad. El contexto relacional que se estableció durante el estudio, permitió que los participantes experimentaran el valor de observar y focalizarse, en detectar, asumir y aplicar su propio talento y el de sus colaboradores a acciones concretas. Una vez se descubrieron los talentos y las fortalezas individuales durante la etapa de diagnóstico se buscó que el mismo individuo las desarrollara a través del coaching y la auto-observación y se hiciera consciente de los beneficios personales de adoptar nuevas conductas, que, si bien eran requeridas por la organización, lo enriquecían personalmente y laboralmente.

El proceso de comunicación como competencia básica para el relacionamiento y el desarrollo social, demostró estar íntimamente asociado al grado de autoconsciencia, de autorregulación, capacidad de contextualización y calidad de la automotivación en los auto-reportes de los participantes. Así mismo, este proceso convirtió el lenguaje en una herramienta conscientemente poderosa para generar una transformación significativa en el ámbito organizacional y personal. A través del coaching, las lecturas, los videos y demás herramientas didácticas los participantes pudieron desarrollar y aplicar habilidades para la comunicación efectiva.

Finalmente, al ser esta una indagación apreciativa, fue posible obtener un conocimiento interno de las fortalezas, logros y motivaciones del grupo de directivos de Terranum y obtener resultados sobre el impacto de la inteligencia emocional en sus habilidades gerenciales. Los resultados en esencial aplican a este grupo de directivos de Terranum; los cuales, fueron elegidos para potencializar de forma personalizada y acorde con la filosofía de la empresa, las habilidades gerenciales necesarias para el óptimo desarrollo del cargo. Es posible que el modelo de intervención tenga resultados similares en una organización que cuente con una estructura y proceso de selección análogas.

Una posibilidad para continuar esta investigación sería buscar como dos competencias, por ejemplo, autoconsciencia y comunicación eficaz, puede incrementarse simultáneamente y mejorar el desempeño de los directivos que lo requieran a través de la utilización de un enfoque apreciativo.

\section{Referencias}

Ardila, A. \& Ostrosky-Solís, F. (2008). Desarrollo histórico de las funciones ejecutivas. Revista de neuropsicología y neurociencias, 8 (1), 1-21.

Beck, A. (1979). Principles of Cognitive Therapy and Emotional Disorders. Boston: Boston University Press. 
Bill George, (2007), True North: Discover Your Authentic Leadership, pg 67-115. New York. Wiley

Bower, J. (1988). El Oficio y Arte de la Gerencia, Vol. II. Escuela de Administración de Empresas de Harvard. Bogotá: Grupo Editorial Norma.

Bradberry, T \& Greaves, J. (2009). Emotional Intelligence 2. San Diego: Talent Smart.

Bushe, G. (2009). Clear Leadership Sustaining Real Collaboration and Partnership at Work (Rev. ed.). Mountain View, Calif.: Davies-Black Pub.

McChesney, C., Covey, S., \& Huling, J. (2016). The 4 disciplines of execution: achieving your wildly important goals. New York: Free Press .Chopra D, \& Tanzi R (2012). Super Brain. New York: Harmony Books

Cialdini, R.B. (2001). Harnessing the Science of Persuasion. HBR 10 MUST READS On Communication (25-42). Boston: Harvard Business Review Press.

Cooper, R.K. \& SawafmA, (1997). Executive EQ. Emotional Intelligence in Leadership and Organizations. New York: The Berkeley Publishing Group.

Cooperrider, D., Whitney D., \& Stavros, J. Appreciative Inquiry Handbook. Bedford Heights, OH: Lakeshore Publishers, 2003, p.14-17.

Cooperrider, D., Whitney D., \& Stavros, J. (2003) Appreciative Inquiry Handbook. Bedford Heights, OH: Lakeshore Publishers, p.14-17.

Curtis, D. (1984). Descartes, Discours de la méthode. London: Grant \& Cutler.

Damasio, A. (2005) El Error de Descartes. La Razón de las Emociones. New York:

Grosset/Putnam Pub.

Damasio, A. (2005). Espinoza avait raison. Joie et tristesse, le cerveau des emotions, Odile Jacon, 2003 (Trad. Cast: En busca de Espinoza (Neurobiología de la emoción y de los sentimientos).

Damasio, A. (2010). Y el cerebro creó al hombre. Barcelona. Destino.

Duggan, W. (2008) Intuición Estratégica. La chispa creativa en la realización humana. Bogotá: Grupo Editorial Norma
Eckman P. (2003). Emotions Revealed: Recognizing Faces and Feelings to Improve Communication and Emotional Life. New York: Times Books.

Eckman P. (2001). Telling Lies: Cues to Deceit in the Market Place. New York: Norton \& Company Inc.

E.H. Kessler, (ed.) Encyclopedia of Management Theory, Sage Publications, 2013.

Fenman, M. (2010). Using Emotional Intelligence at Work: 17 Tried and Tested Activities for Understanding the Practical Applications of Emotional Intelligence.

Fisher, R. \& Shapiro, D. (2005). Beyond Reason. Using Emotions as You Negotiate. New York: Penguin Group

Frijda, M.A. (1988). The laws of Emotion. Blackwell publishers: New York

George, B. (2007). True North. San Francisco: Jossey Bass Pub

Frisch, Ml, Lee, J.R, Metzger, K.,Robinson, J. \& Rsemarin, J. (2012). Becoming an Exceptional Executive Coach. United States of America: AMACON

Gallwey, T. (2009). The Inner Game of Stress. New York: Random House.

Gladwell, M. (2008). Outliers: The story of success. Boston: Little Brown And Company.

Gladwell, T. (2000). Outliers: The Inner Game of WORK. New York: Random House.

Goleman, D. (2006). Inteligencia Social. Mexico: Planeta.

Goleman, D (2002). Vital Lies, Simple thruths. New York, Simon\& Shulter pg.244-251)

Goleman, D. (2003). Emociones Destructivas: Un diálogo científico con el Dalai Lama. Bogotá: Vergara Editores.

Goleman, D. (2002). El Líder Resonante Crea Más. España: Plaza y Janes.

Goleman, D. (1995). Emotional Intelligence. Why it can matter more than I.Q. New York: Bantan Books

Goleman, D. (1998). Working With Emotional Intelligence. New York. Bantam Books.

Goleman, D., Kaufman, P, \& Ray, M. (2002). El Espíritu Creativo. Argentina: Vergara Editores. 
Goleman, D. (2012) El Celebro Y La Inteligencia Emocional. Nuevos Descubrimientos, España. Ediciones B.S.A.

Goleman, D. (2013). FOCUS. The Hidden Driver of Excellence. N. York, Happer Collins.

Goleman, D. (2015). What Makes a Leader? HBR 10 must reads, On Emotional Intelligence $(1-24)$. Boston: Harvard Business Review Press.

Goleman, D. Boyatzis, R., \& Mckee, A. (2015). Primal Leadership: The hidden driver of great performance. HBR 10 must reads, On Emotional Intelligence (25 43). Boston: Harvard Business Review Press.

Grant, A (2014). Give and Take: Why Helping Others Drives our Success. New York. Penguin.

Graetz, F. (2002). Strategic Thinking versus Strategic Planning: Towards Understanding the Complementarities. Management Decisions, 40(5/6), 456-462.

Hammond, S. (1998). The thin book of appreciative inquiry (2nd ed.). Plano, Tex.: Thin Book Pub.

Hammond S.A (2013) The thin book of appreciative Inquiry. Thin Book Publishing.

Harre, R, \& Gerrod, P. (2002). The Emotions. Social, Cultural and Biological Dimensions. London: Sage Publications.

Hillman, J. (2013). Archetypal Psychology. Uniform edition Screen Publications. New York.

Hume, D. (1739) Citado en junio 12, El espectador, Francisco Gutiérrez

Johnson, R., \& Eaton, J. (2001). Coaching successfully. New York: Dorling Kindersley Pub.

Jyotirmayananda (2006) The art of Positive Thinking. Miami: Yoga Research Foundation.

Krishnamurti,J. (1984). The Flame of Attention. New York: Harper \& Row

LeDoux, J (1999) El cerebro emocional. Madrid. Ed. Planeta.

Katzenbach Jon R., Smith Douglas K. The discipline of teams - HBR. April 1993.

Kinni, T (2003) The art of appreciative inquiry. En: "Exploit What You Do Best," Harvard Management Update, Vol. 8 , No. 8.
Krizan, A.C., Merrimer, P., Logan, J., \& Williams, K. (2008). Business Communication. Eighth Edition. Canada. Nelson Education.

Levenson, R.W. (1994). Human Emotion. A Functional View. In P. Ekman \& R.J. Davidson (Eds). The nature of Emotions: Fundamental Questions (pp. 123-126). New York: Oxford University Press.

Lipton B.H (2005) The Biology of Belief. California: Hay House.

Loweney, C. (2004). El liderazgo al estilo de los Jesuitas. Bogotá: Grupo Editorial Norma.

Lynn A.B. (2002). The Emotional Intelligence Activity Book. New York: AMACOM

Martín L.S. (2013). Neurociencia, empresa y marketing. Madrid. ESIC.

Maxwell, John C. (2009). How successful people think. New York: New York Times.

Mertz, D.(2004). Dursley Duplicity: The Morality and Psychology of Self-Deception. En "Harry Potter and Philosophy- If Aristotle Ran Hogwarts" (22-37). Chicago: Carus Company Pub.

Mersino, A. (2007). Emotional Intelligence for Project Managers. Part 2: Project Management Begins With Self-Management (33-70) New York: AMACOM

Meyer, E. (2015). Getting to Sí, Ja, Oui, Hai, and Da. HBR 10 must reads 2017. (105-116) Boston: Harvard Business Review.

Mind Tools, 2012. Test Manejo del tiempo. Traducción Libre, C. Salgado (2012). http://www.mindtools.com/ pages/main/newMN_HTE.htm

Mongomery, C. (2012). El Estratega. Conviértete en el Líder que tu Negocio Necesita. Bogotá: Distribuidora y Editorial Aguilar.

Moya-Albiol L, Herrero N. \& Bernal C. (2010). Bases Neuronales de la Empatía. Rev Neurol 2010; 50: 89100 .

Murphy, J.D. (2005). Flawless Execution. Use the techniques and systems of America's fighter pilots. New York: Regan Books.

Nash, J,F. (1950). The Bargaining Problem. Econométrica, 18 (2), 155- 162.

Olson, A. K, Simersonm B. K. (2015). Leading with Strategic Thinking. Four Ways Effective Leaders gain 
insight, drive change and get results. New Jersey: Wiley \& Sons.

Pease, A. (1997) Body Language. London: Sheldon Press.

Perlow L., Williams S. (2013) Is Silence Killing your Company? HBR 10 must reads On Communication (91-104) Boston: Harvard Business Review press.

People Performance International (2015) Metodología DISC + Encontrado en http://people-performance. com/metodologias/metodologia-disc

Ponti F. (2007) Caminos de la negociación. Verticales de Bolsillo.

Quillian, S. (2004). Body Language. Buffalo, New York: Firefly Books.

Rath, T. (2007) Strengths Finder. New York. Gallup.

Ries, A, T., Trout, J (1981). New York: Warner Books.

Ruelas-Gossi, A \& Sull, Donald N. (2006). Orquestación Estratégica: la clave para la agilidad en el escenario global. Harvard Business Review. América Latina.

Salovey, P., \& Mayer, J. (1990). Emotional Intelligence. Imagination, Cognition and Personality, 9, 185-211

Segal J. (2008) The Language of Emotional Intelligence. New York. McGraw Hill

Sorensen, P \& Cooperrider, D. (2000). Appreciative Inquiry. Illinois: Stipes Publishing L.L.C

Salgado C. (2014) Como manejar estados emociones perturbadores

Salgado C. (2014) Anotaciones sobre como predisponerse hacia el logro.

Salgado C (2014) Guía para una comunicación eficaz

Salgado C. (2012) Cómo aplicar las competencias emocionales para manejar las presiones de la vida laboral.

Salgado, C. (2010). Cartilla de Inteligencia Emocional Aplicada a la Gestión Comercial. Bogotá: Confinanciera.

Salgado, C. (1991). El modelo de Milán: Aspectos básicos y reflexiones sobre su empleo en nuestro medio. Revista de la Fundación de Psicoanálisis y de las Psicoterapias. 24-36.

Salgado, C. (1988). Comunicación y psicoterapia. Revista Latinoamericana de psicología. 20 (3). 369-384.
Sood, Amit, M.D. (2013). The Mayo Clinic Guide to Stress Free Living. Mayo Clinic. United States of America: DaCapo Press.

Thomas, C. (1998). El Arte de la Guerra Ilustrado. Sun Tzu. Madrid: Edaf.

Waytz, A. (2016). The limits of Empathy. Harvard Business Review. HBR 10 must reads. P 117.

Whetten, D.A\& Cameron, K (2011). The critical Role of Management Skills. Developing Management Skills. (3-23) New Jersey: Prentice Hall.

Goleman, D. (2009) ¿What Makes a Leader? HBR 10 must reads on Emotional Intelligence (1-22). Boston: Harvard Business Review press.

Williams G.A., Miller R.B. (2013). Change The Way You Persuade. Harvard Business Review. HBR 10 must reads On Communication. $(1-24)$ Boston: Harvard Business Press. 